Pain in children with severe intellectual disability:

\title{
A Guide for Parents
}

\section{Contact us}

General enquiries: 01267244200

Email: info@cerebra.org.uk

\section{Postal address}

Cerebra

2nd Floor Offices, Lyric Buildings

King Street

Carmarthen

SA31 1BD.

\section{Website}

http://w3.cerebra.org.uk

The findings of this report are those of the author, not necessarily those of Cerebra.

Registered Charity no. 1089812

Company no. 4336208

First published 2015

Review date 2018

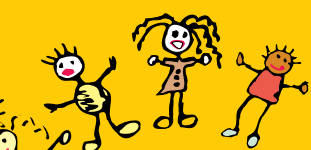

Cerebra $0.52 a s$
This guide aims to help the parents of children with severe intellectual disability and/or communication difficulties understand how pain may affect their child.

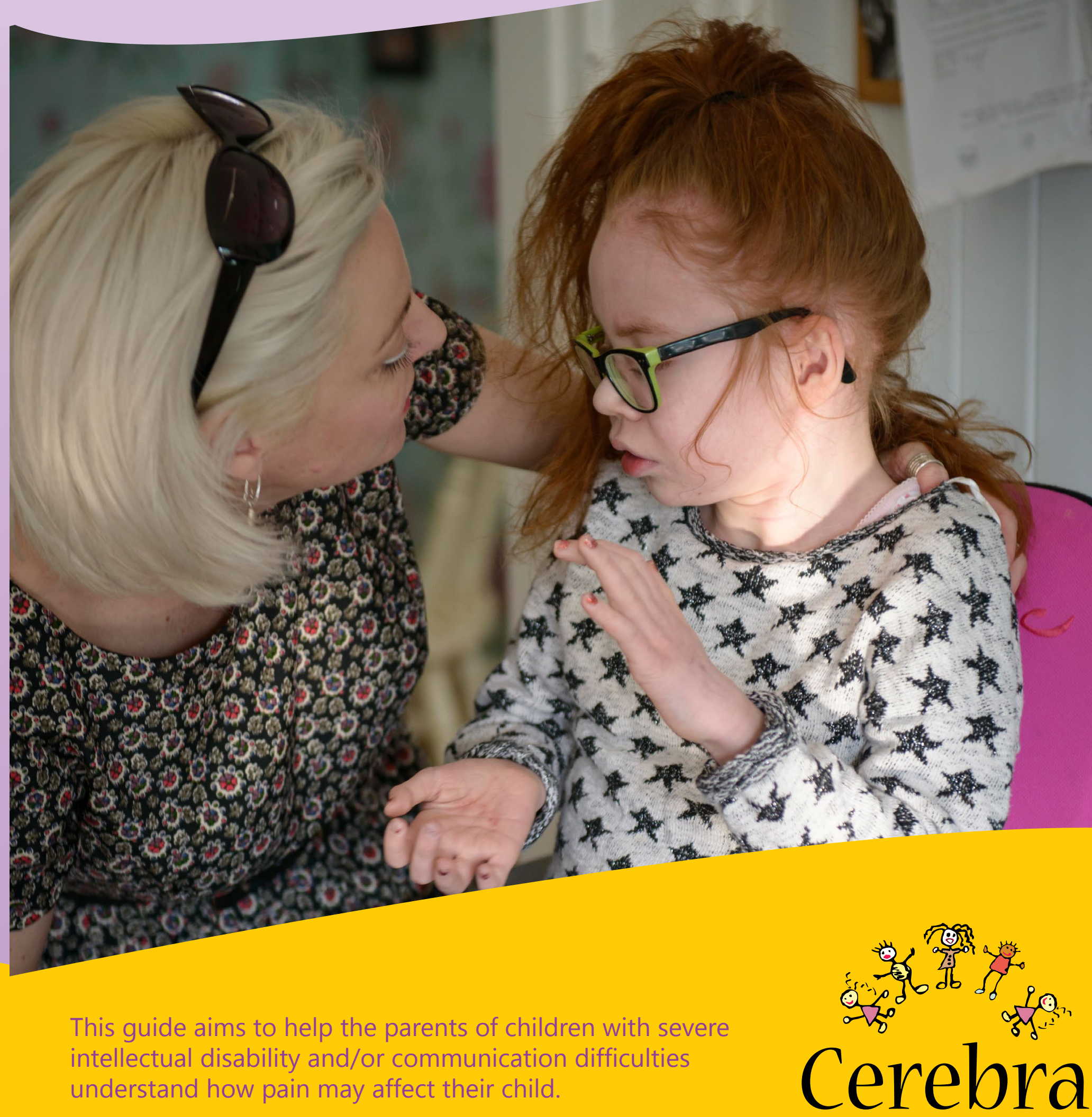

Positively Different 
As a national charity, Cerebra strives to improve the lives of children with neurological conditions through research, education and direct, on-going family support. Living with neurological conditions can make life very hard, not just for the child but for their family too. Through the generosity of our supporters we are able to make it a little easier.

This guide is provided free of charge but if you would like to make a donation to help cover the costs of research and updating, it would make a huge difference. Please scan here to make a donation.

\section{可化 \\ . \\ .

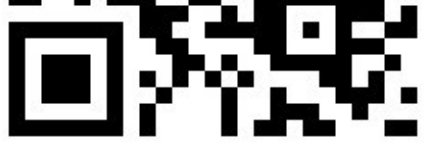

To donate by text send CERE12 and then the amount to 70070, or telephone our Fundraising Department on 01267244221.

Report by Prof. Chris Oliver and Dr Lucy Wilde, Cerebra Centre for Neurodevelopmental Disorders, School of Psychology, University of Birmingham, and Dr. Kate Eden, University of Bath.

Reviewed by Dr. Caroline Richards, Cerebra Centre for Neurodevelopmental Disorders, School of Psychology, University of Birmingham. 


\section{Pain in Children with Severe Intellectual Disability: A Guide for Parents}

\section{Introduction}

This guide will help the parents of children with severe intellectual disability and/or communication difficulties understand how pain may affect their child.

It does so by explaining possible causes of pain in children with intellectual disability, presenting information about how pain might be shown by children who cannot tell us they are in pain and discussing the effects of untreated pain.

- A question and answer format is used to present this information

- Detailed illustrations of key points are provided in the 'Focus on...' sections

- The Resources section includes links to other briefings and tools that you can access

- The References section at the end of this guide provides the details of the research which informs this guide

- The Glossary provides explanations of commonly used terms which are highlighted in bold in the text

- The 'Quick index' on the next page signposts you to the pages where you can find information on specific topics in this guide.

This guide is part of a series of guides published by Cerebra that aim to give parents of children with disabilities and/or special educational needs information on how to get the help and support they need. This series of guides can be found on the Cerebra website: http://w3.cerebra.org.uk 
Importance of identifying pain

Pg.3

Causes of pain in children with intellectual disability

Pg.4

Focus on: Gastro-oesophageal reflux

Pg.6

Focus on: Health conditions in specific syndromes and disorders

Pg.7

What child behaviours are associated with pain?

Pg.10

How can we try to assess whether a child is in pain?

Pg.11

What evidence is there that pain might cause self-injury?

Pg.14

How can I tell what is causing my child's pain?

Pg.16

Focus on: Pain and self-injury

Pg.17

Is the importance of identifying and treating pain in children with

Pg.19 intellectual disability widely recognised?

Glossary

Pg.20

Resources

Pg.22

References

Pg.23 


\section{The importance of identifying and treating pain}

\section{Difficulties identifying pain}

Pain is difficult to identify in children with intellectual disability and/or communication difficulties. It is a subjective, personal experience and the gold standard for describing and assessing pain is self-report. ${ }^{1}$ Often, this is not possible in children who have severe or profound intellectual disability or communication difficulties. Identifying pain is made more difficult by the fact that differentiating between internal states can be difficult for less able children, even those with mild to moderate intellectual disability. A common example of this is where children describe having stomach or back pains but are actually experiencing anxiety.

\section{Increased risk of pain}

Problems with pain identification are particularly concerning because children with intellectual disability have higher rates of health problems associated with pain and discomfort., Examples of such illnesses include respiratory disease, ${ }^{4}$ dental problems, ${ }^{5,6}$ and epilepsy. ${ }^{7}$ This increased risk of potential health problems demonstrates how important it is that everyone can identify pain and discomfort in children with intellectual disability.

\section{Accessing appropriate treatment}

Difficulty identifying and assessing pain and poor health is likely to contribute to underdiagnosis of medical conditions, leading to continued pain and discomfort. 8 .9 Ultimately, improving understanding of pain may lead to better healthcare and help to address the health inequalities faced by children with intellectual disability. ${ }^{10}$

\section{Reducing associated behaviour problems}

Untreated pain may also be associated with increased risk of developing behaviours such as self-injury (see Glossary section on page 21) and aggression. ${ }^{11}$ You can find further detailed information about self-injury in the Cerebra briefing 'Self-injurious Behaviour in Children with an Intellectual Disability', (see Resources section on page 22).

\section{Summary}

Identifying and treating pain relieves discomfort, addresses underlying health problems and reduces specific risk factors for problematic behaviours - all outcomes likely to enhance quality of life. 


\section{Questions and answers}

\section{What might be the causes of pain in children with intellectual disability?}

There a number of possible causes of pain in children with intellectual disability. These include increased risk of health problems, self-injurious behaviour (see Glossary section on page 21) and discomfort where the cause of the intellectual disability is also related to a physical disability limiting mobility.

\section{Health problems}

Some health problems are more common in children with intellectual disability and suggest an increased likelihood of pain. ${ }^{12,13,14}$ These include:

- Tooth decay

- Gastro-oesophageal reflux disorder (reflux or heartburn) ${ }^{15,16}$ (see Glossary section on page 20)

- Middle ear infections (also known as otitis media)

- Constipation.

In addition to specific health problems that are more likely in children with intellectual disability, some conditions which cause intellectual disability are associated with a wide range of specific health problems which indicate a very strong need to monitor for possible signs of pain.

Reflux is more common in certain genetic syndromes and disorders, including Cri du Chat Syndrome, Angelman Syndrome and Cornelia de Lange Syndrome ${ }^{17,18,19,20}$ and in Autism Spectrum Disorders. ${ }^{21}$ We also know that the risk of developing diabetes mellitus is increased in Prader-Willi Syndrome ${ }^{22}$ and seizures are common in children with cerebral palsy.

If your child has a syndrome or disorder that is associated with increased risk for particular health problems it is important that health professionals and support staff involved with your child's care are vigilant to the development of these difficulties.

\section{Discomfort}

Some causes of intellectual disability are also related to physical disability. Where a disability affects mobility, children may be unable to make adjustments to their surroundings to address things which are causing them discomfort. Where communication is limited this may also mean that children are unable to request adjustments needed to reduce discomfort. If the causes of discomfort are left unaddressed they may lead to pain.

Things which may lead to discomfort include:

- Poor posture when sitting

- Pressure caused by sitting or lying in one position for too long

- Rubbing clothing

(See Resources section on page 22 for more information about postural care from Mencap and guidelines for the prevention and management of pressure sores). 
It is important to ensure that your child is comfortable in their environment and that causes of discomfort from this source have been ruled out if a child is showing signs of pain.

\section{Self-injury}

Children with intellectual disability are at increased risk of developing self-injurious behaviours (such as head banging or self-biting), with rates of $4-10 \%$ reported. ${ }^{24,25}$ There are certain child characteristics that are specifically associated with increased risk of self-injury. ${ }^{26}$

These include:

- A greater degree of intellectual disability 26,27

- High levels of activity \& impulsivity ${ }^{28,29,30}$

- Autism Spectrum Disorder (see Glossary section on page 20) $31,26,32$

- High levels of repetitive \& stereotyped behaviour (see Glossary section on page 21$)^{28,33}$

- Poor communication skills 34,26

- Low mood 35,36

A number of genetic syndromes are also associated with increased risk of self-injury, ${ }^{28}$ for example in Smith-Magenis syndrome, self-injury can be particularly likely (shown by up to $90 \%$ of people with the syndrome). ${ }^{37,38,39}$

An obvious consequence of self-injury can be damage to the skin, such as bruising or cuts and abrasions which may result in pain. Severe self-injury can result in bone trauma and fractures. A range of self-injurious behaviours may result in such damage, including skin picking, skin pinching, self-hitting, self-biting and head banging. Behaviours such as head banging or eye poking can also result in discomfort without a visible indication of damage, such as headaches.

However, there is quite a complicated relationship between pain and self-injury - while it seems clear that self-injury would result in pain there is also convincing evidence that experiencing pain increases the risk of a child showing self-injury. This will be discussed in more detail subsequently, for now it is important to emphasise the following:

If a child is both showing self-injury and appearing to be in pain it is not necessarily the case that the pain is being caused by the self-injury, there may be another underlying cause of the self-injury such as an untreated health problem.

More information about self-injury can be found in the Cerebra briefing 'Self-injurious Behaviour in Children with an Intellectual Disability', and on the Further Inform Neurogenetic Disorders (FIND) website (under 'key topics'), see Resources section on page 22. 


\section{Focus on: Gastro-oesophageal reflux (reflux)}

As noted previously, gastro-oesophageal reflux is a common cause of pain in children with intellectual disability. This section will give more information about this condition and how it might affect children with intellectual disability.

What is it? Gastro-oesophageal reflux, sometimes called gastro-oesophageal reflux disease (GORD) or just reflux is where stomach acid leaks out up into the oesophagus (the tube from the mouth to the stomach). It can cause a range of symptoms including heartburn (burning pain in the chest or discomfort after eating), acid reflux (causing an unpleasant taste in the mouth), pain and difficulty with swallowing.

Who is at risk of developing it? Around $50 \%$ of people with profound intellectual disability will develop reflux, compared to $10 \%$ to $20 \%$ in the general population. ${ }^{15}$ In people with an Autism Spectrum Disorder it is reported to be even more common (occurring in up to $70 \%$ of people). ${ }^{21}$ High rates have also been described in genetic syndromes associated with intellectual disability. For example over $90 \%$ of individuals with Cornelia de Lange Syndrome have reflux. ${ }^{18,19}$ Other characteristics such as being overweight can also increase the likelihood of developing reflux. ${ }^{40}$

What causes it? The most common cause is problems with the muscle at the bottom of the oesophagus (the lower oesophageal sphincter). This ring of muscle is like a valve, which opens to allow food to fall into the stomach, then closes stopping the stomach acid from leaking out. If this valve does not close properly acid can leak out of the stomach causing the symptoms described above.

How is it diagnosed? In people who can verbally communicate, doctors diagnose reflux from their account of their symptoms. Where people cannot report this, there are other signs that may indicate reflux in addition to more general signs of discomfort. Possible signs include: ${ }^{41,42}$

- Arching his/her back

- Bad breath

- Scratching/rubbing chest

- Grinding teeth

- Coughing (particularly at night)

- Refusing food

- Excess salivation

- Gagging/regurgitating/vomiting

- Night waking

- Tooth decay

- Hoarse voice

- Frequent respiratory tract infections (see Glossary on page 21)

(For more information about signs of reflux and NHS patient information, see Resources section on page 22).

These signs do not definitely indicate that a child has reflux; many of these signs may be shown in the absence of reflux. 
One way that doctors can check to see whether reflux is occurring is through a procedure called an endoscopy, where a camera on a thin, flexible tube is swallowed to let doctors look at the oesophagus to check for damage from acid.

How is it treated? There are a range of possible treatments that doctors might explore. Antacid medications work to neutralise the effects of the stomach acid (e.g. Gaviscon liquid). A different type of medication that can be used is one which reduces stomach acid production (e.g. Omeprazole). Other possible treatments include an operation called a fundoplication which tightens the muscle to stop the stomach acid escaping. If caregivers are concerned that their child has reflux they should discuss this with their GP.

\section{Key points}

- Risk of this painful condition is increased in children with intellectual disability.

- Not being able to communicate means that the usual ways that a doctor would identify reflux are not always available.

- There are specific behaviours and signs, in addition to general indicators of pain (see 'What child behaviours are associated with pain?' on page 10), that might indicate a child has this health problem.

- Once identified and treated using the options described, this pain and discomfort should reduce. This may lead to a reduction in other pain related behaviours, including self-injury if this is pain related (see Alice's story on page 17).

(More information about gastro-oesophageal reflux from the NHS can be found in the Resources section on page 22).

\section{Focus on: Health conditions in specific syndromes and disorders}

There are some syndromes and disorders which are associated with particular health issues. This section will focus on how knowing that a child has a specific syndrome or disorder might inform how we think about their experiences of pain. The Further Inform Neurogenetic Disorders (FIND) website (See Resources section on page 22) has detailed information about a number of different genetic syndromes including Cri du Chat syndrome, Cornelia de Lange syndrome and Angelman syndrome.

Causes of intellectual disability: Some intellectual disabilities are caused by specific syndromes or disorders. Syndromes can include genetic syndromes caused by changes in genetic information. These include well known syndromes such as Down syndrome and fragile $X$ syndrome and also rarer syndromes including Cornelia de Lange syndrome, Cri du Chat syndrome and SmithMagenis syndrome. See the Cerebra briefing 'Is the diagnosis of a genetic disorder important for children with intellectual disability?' (See Resources section on page 22) for more information about how understanding specific features of a genetic syndrome may be helpful for caregivers and professionals. 
Other syndromes and disorders can be caused by environmental influences as the child is developing in the womb, such as foetal alcohol spectrum disorder or problems during birth, e.g. cerebral palsy. Knowing your child has a specific syndrome or disorder can provide valuable information about possible health problems.

We will consider the example of Tuberous Sclerosis Complex to demonstrate how increased understanding of the syndrome as a whole can improve identification, treatment and prevention of pain.

Health problems and pain: Tuberous Sclerosis Complex is a disorder associated with dysregulated cell growth, and can cause tumours to grow in lots of different parts of the body. ${ }^{43}$ This includes the brain (causing raised pressure in the brain leading to headaches), kidneys (causing pain, bleeding and renal failure) and heart (causing chest discomfort). ${ }^{44,45,46}$ Epilepsy is also common and may lead to discomfort when experiencing seizures. ${ }^{47}$ Given the complexity of health problems associated with this disorder it is very likely that children and adults with this syndrome may experience pain and discomfort at some point.

A significant proportion of people with Tuberous Sclerosis Complex (30\%) have profound intellectual disability ${ }^{48}$ and are likely to have communication impairments. Because of this, they are unlikely to be able to report pain caused by their health problems. They may therefore be in significant discomfort if painful health problems are not detected by routine monitoring, reducing their well-being. Health problems going unnoticed may also lead to a lack of treatment for potentially life-threatening illnesses, such as brain tumours, which can block the drainage of fluid from the brain.

Monitoring for pain: Published clinical guidelines ${ }^{49}$ recommend that people with Tuberous Sclerosis Complex are closely monitored, with regular tests for the development of tumours and other health problems.

Caregivers also need to be particularly vigilant for signs of pain and ill-health in their child. This involves working with health professionals to pursue monitoring and treatment for conditions that are likely to develop in their child.

In addition to this, of course, any changes in behaviour suggesting pain/discomfort (see 'What child behaviours are associated with pain?' on page 10) should be investigated, as common conditions that are not specifically related to the syndrome (ear infections, reflux, constipation) may be involved. 


\section{Claire's* story}

Below is a quote from a parent of a child with Tuberous Sclerosis Complex who took part in a research study where observations of behaviours identified high levels of pain related behaviours. Claire's caregivers were told about these results in a feedback report from the researchers.

"Claire's individual results came back to us having been analysed and several times in this report it recommended that we check Claire out with her GP as she was showing behavioural signs of being in pain. We thought these behaviours were part of her TS and challenging behaviour in general. In fact during discussions, her renal consultant decided to push for an MRI scan as Claire's paediatrician locally had not felt it necessary to arrange one before this. Claire was then found to have a SEGA [type of brain tumour] which was removed by emergency surgery 2 weeks after getting the MRI results. So indeed her behaviours were an expression of pain that no one knew about because of her communication difficulties."

*Name changed

Here Claire was showing signs of pain related behaviour which could easily be seen as just part of the syndrome. Her mother worked with Claire's medical team to pursue monitoring for a known likely health problem associated with her daughter's condition, with a positive outcome for Claire.

\section{Key points:}

- For children whose intellectual disability is caused by (or associated with) a particular syndrome or disorder, those involved in their care should be aware of the range of known health problems associated with the disorder that might cause pain and where the cause of this pain may be.

- There may be clinical guidelines for the care of children with certain conditions which outline the monitoring and tests that your child should be receiving.

- Any changes in behaviour indicating pain should be followed up, even if causes related to health problems directly related to your child's syndrome or disorder have been ruled out. 


\section{What child behaviours are associated with pain?}

Two broad areas of everyday behaviour have been shown to be strongly associated with health and pain; low mood and levels of activity.50,51,52 Two of the most commonly used measures to assess pain in those who cannot communicate, are the Face, Legs, Activity, Cry, Consolability (FLACC) measure ${ }^{53}$ and the Non-Communicating Children's Pain Checklist-Revised (NCCPC-R) measure. ${ }^{54}$ Both involve assessing an individual's mood and how active they are. These specific measures are discussed in detail in the next section, 'How can we try to assess whether a child is in pain?' (see page 11).

Mood: People with intellectual disability who have health problems are described by caregivers as having significantly lower 'mood' compared to individuals without health problems. ${ }^{50}$ This low mood may be associated with increased negative affect (more negative facial expression, crying). Crying, screaming and sobbing are included in the FLACC measure as behaviours potentially related to pain, as is the extent to which the person can be consoled, reassured or distracted. ${ }^{53}$ Similarly the NCCPC-R includes moaning, crying or screaming together with facial expressions, including turned down mouth and pouting, as pain related behaviours.

However, low mood is not just signalled by facial expression and vocalisations (see Glossary section on page 21); it may also be indicated by reduced interest in or engagement with day to day activity and reduced pleasure in activities that were previously enjoyed.

Activity: High activity levels have also been linked to painful health problems. For example 'hyperactivity' has been found to be more characteristic of children who are experiencing a health condition (reflux), than 'typical' symptoms of that condition (vomiting or regurgitation). ${ }^{19}$ Squirming, shifting back and forth and jerking are specific types of activity that have been related to pain and are included in the FLACC measure. ${ }^{53}$ In the NCCPC-R high levels of activity such as jumping around, being agitated or fidgety is recorded as a possible sign of pain. Importantly however, being less active, not moving or being quiet are also recorded as behavioural indicators of pain in this measure. ${ }^{54}$ This suggests that attention should be paid to changes in activity levels or to extremes in activity levels being shown (either over activity or under activity).

As well as low mood and high activity, less common and more specific difficult behaviours are also related to poor health. Self-injury, aggression, property destruction (see Glossary section on page 20), stereotyped behaviour (see Glossary section on page 21) and tantrums have all been found to be increased in children with intellectual disability when they were sick (with an observable or measurable sign of illness, such as diarrhoea or a temperature) compared to when the same children were well. ${ }^{55}$ More information about pain and self-injury, aggression and property destruction is covered in the answers given to the following questions: What evidence is there that pain might cause self-injury? How might pain be linked to self-injury? How might pain be related to other challenging behaviours? (pg. 14-16). 
Finally, physiological changes such as changes in appetite (eating less or lack of interest in food) and sleeping (increased or decreased sleeping) have also been associated with pain. ${ }^{56}$

\section{How can we try to assess whether a child is in pain?}

Sometimes it seems clear when a child is in pain, for example if they hurt themselves and cry immediately afterwards. Other times, children's responses to pain are not as clear (e.g. a child showing no immediate response to hitting their head but developing a headache later) or the pain may be more chronic (e.g. long term pain from reflux) and so less easy to relate to a particular episode. In these situations having specific ways to assess pain may be useful.

Self-report: Self-report of pain is often assessed in children using visual scales including ${ }^{57}$

- Faces which range from smiling or neutral (no pain) to frowning/grimacing (lots of pain) to which children point.

- Lines representing the extremes from 'no pain' to the 'most pain' along which children indicate where their pain is.

- Objects which increase in size to represent increasing pain, from which children choose.

Children over 3 years old may be able to reliably report pain using such tools ${ }^{57}$ and self report of pain has been used successfully with some individuals with intellectual disability. ${ }^{58}$ For example, it has been demonstrated that a group of children with mild intellectual disability were able to self-report level of pain using simple facial scales and number scales, or with the use of words such as 'a lot'. ${ }^{2}$ Other researchers however have found that when these types of self-report scales are used with children with intellectual disability ratings seem to be less reliable. Even when scales were modified to make them simpler, about $50 \%$ of children with mild to moderate intellectual disability were not able to effectively give a rating of pain using a range of different types of self-report measures, including those described in the list above. ${ }^{59}$ It has therefore been suggested that self-report measures that are currently available probably should not be used as the first method employed to assess pain in children who cannot communicate. ${ }^{60}$

Given the potential limitations of self-report for children with intellectual disability, we can instead try to infer pain from our observation of children's behavioural indicators of pain.

Caregiver/professional observations: Some observation measures rely on very specific observation of facial expressions (e.g. Facial Action Coding System ${ }^{61}$ ), whereas others focus on broader behaviour patterns such as body movements, emotional responses and changes to physiological patterns such as eating and sleeping routines. Two of these measures which are widely used to evaluate pain in non-communicating children in both clinical and research situations are described on the next page. 
The FLACC (Face, Legs, Activity, Cry, Consolability) ${ }^{53}$ measure has five subscales. Each subscale describes a collection of behaviours which may indicate pain.

A score of zero on each of the subscales refers to no pain-related behaviour (e.g. face subscale includes 'no particular expression or smile') and a score of two on each subscale, the maximum score, includes behaviours which are indicative of high levels of pain (e.g. face subscale includes 'Frequent to constant quivering chin, clenched jaw'). The score on each of the subscales are summed to give a total between zero and ten, zero reflecting no or low level of behavioural indicators of pain and a higher score representing a higher number of behavioural indicators of pain.

The FLACC is able to reliably identify pain in children with intellectual disability (two different people are likely to rate behaviours similarly), and scores from FLACC observations have been found to be similar to those on both a different pain measure completed by parents and selfreport ratings made by children who are able to self-report. Finally, FLACC scores decrease after painkillers are given. Together this suggests that this observation measure is a promising tool for assessing pain in children who cannot self-report..$^{53}$

\section{The FLACC Pain Scale}

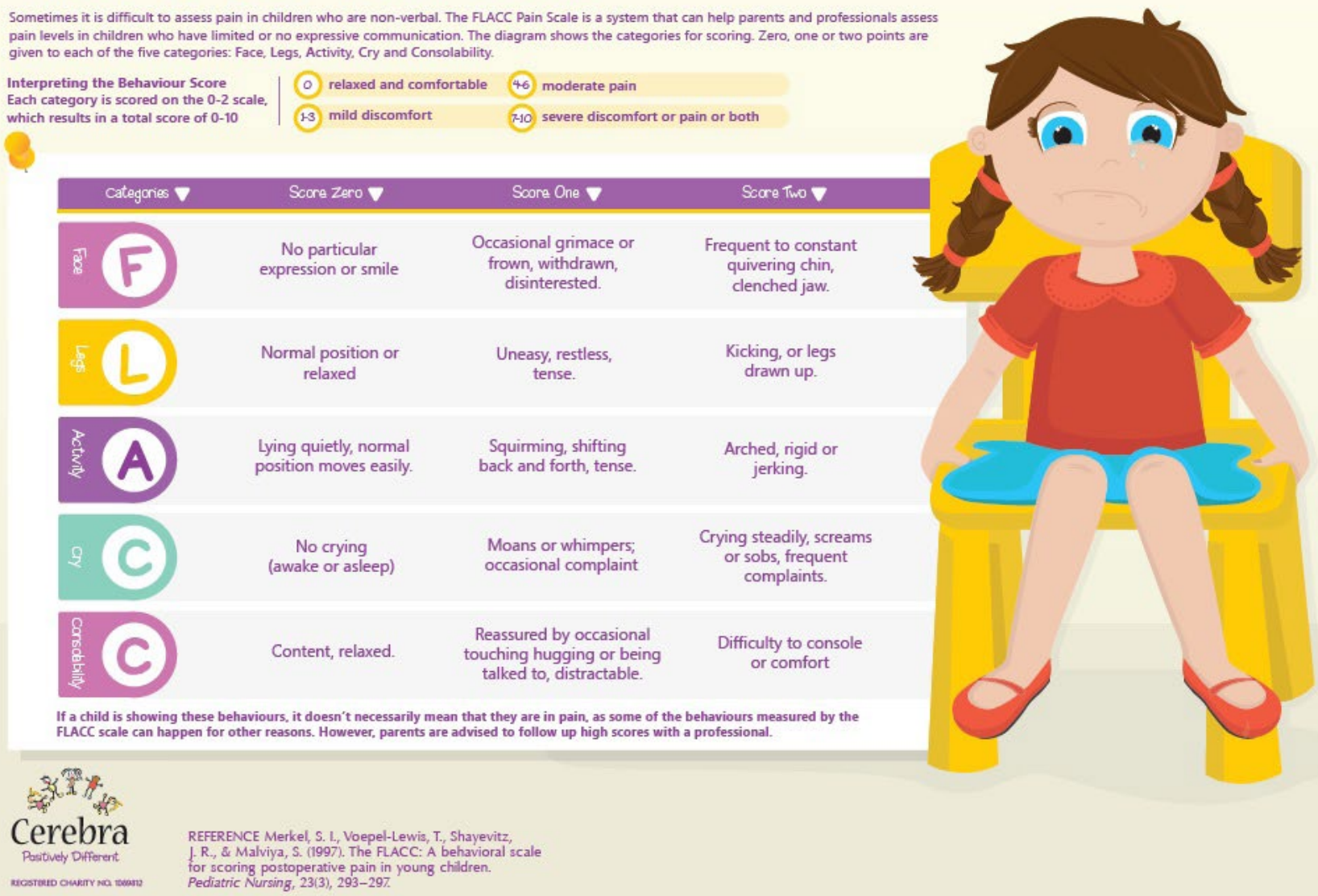

An example of the FLACC tool for assessing pain-related behaviour (see Resources section on page 22). 
Another very widely employed observational measure of pain is the Non-Communicating Children's Pain Checklist- Revised (NCCPC-R). ${ }^{62,54,13,63,64}$ The NCCPC-R is a 30 item questionnaire, which asks the person completing it to rate how often behavioural indicators of pain are shown by a person over a two-hour period. This scale was originally designed to measure postoperative pain but has been revised to be used in the home.

Behaviours that are asked about include facial expressions, activity and vocalisations. In addition to this it asks about social behaviour (e.g. being irritable or withdrawn and seeking comfort), interest in food and sleeping patterns and physical signs such as shivering, sweating, gasping or paleness. People completing the questionnaire are asked to say whether these have been shown very often, fairly often, just a little, not at all or to say if it is not applicable. As with the FLACC a higher score represents more behavioural indicators of pain.

The NCCPC-R has been found to successfully identify behavioural indicators of pain. When caregivers completed the measure for children with intellectual disability scores on this measure were higher at times when children were known to be in pain (e.g. caused by injury, a chronic condition, illness or a medical procedure) than when they were not in pain. ${ }^{56}$

This suggests that this tool may also be effective in identifying pain/discomfort in this group of children.

We can see that both widely used tools try to measure aspects of the general behaviours discussed previously including mood and activity. The tools ask caregivers to watch their child for a certain amount of time and rate the behaviours that they see. While a high score on this type of measure does not mean that a child is definitely in pain it can be used as an indication that this might be an issue, particularly if considered together with other information including general health and challenging behaviour.

\section{How could I use these measures?}

If you suspect that your child is in pain you may want to use some of the approaches to assessing pain described above. There are a number of different ways this may happen.

Helping your child to tell you: If your child is able to make the relation between their feelings and pictures (for example if they seem to be able to do this using communication aids such as PECS (Picture Exchange Communication System) or Makaton (see Glossary section on page 20) then you may want to explore using visual rating scales e.g. the type of face rating scale described previously. Using these when you know your child is well and ill may provide a baseline (see Glossary section on page 20) for future use, when you are unsure if they are feeling unwell or how unwell they are feeling.

Helping to recognise your child's specific pain behaviours: It may also be useful to start to familiarise yourself and others with your child's 'pain signature' (see Glossary section on page 20) - the unique pattern of behaviours that they show when they are in pain. 
You can do this easily by using a mobile phone to record your child's behaviour when you know they are experiencing pain. This can then serve as a record of the behaviours to watch out for in future that may indicate pain and be used to show health professionals the types of behaviours that lead you to believe they are experiencing pain. An example may be recording your child pulling their ears when they have an ear infection.

Helping you to record and measure possible pain: If your child is not able to tell you that they are in pain or use pain scales then observing their behaviour for indicators of pain may be useful. Links to the FLACC tool described previously, together with instructions on how to use it to rate behaviours when you think your child is in pain are available (see Resources section on page 22).

\section{What evidence is there that pain might cause self-injury?}

As noted previously, it seems intuitive that self-injury might cause pain but perhaps less so that pain might cause self-injury. There is good evidence that these two things tend to co-occur.

Observational measures of pain show significantly higher numbers of pain related behaviours in people with self-injury compared to those without this behaviour. ${ }^{13,63,64}$ However this does not show cause, as self-injury may be causing pain as well as the other way around.

More convincing evidence that pain might result in self-injury comes from the fact that behaviours such as self-injury and aggression increase when children are ill with high temperatures or upset stomachs compared to when they are well. ${ }^{55}$ This suggests that the pain/ discomfort caused the behaviour, as it is not likely that the self-injury could cause these types of illness (in contrast to pain from an injury).

Finally, further evidence is provided by reductions in challenging behaviour following treatment for painful health conditions ${ }^{12,11}$ and behaviour fluctuating with the improvement and deterioration of health. ${ }^{13}$

Increasing evidence points towards there being a causal relationship between experiencing pain and going on to show self-injury. If a child is showing self-injury and causes (or 'functions', see Glossary section on page 20) are not clearly identified then it is important to examine pain as a possible cause.

More information about self-injury can be found in the Cerebra briefing 'Self-injurious Behaviour in Children with an Intellectual Disability' and on the Further Inform Neurogenetic Disorders (FIND) website (under 'key topics'). See Resources section on page 22. 


\section{How might pain be linked to self-injury?}

It might seem odd that a response to pain could be to cause more pain. Several explanations have been suggested as to why this might be the case, including those presented below. We do not know for certain which of these may provide the best explanation of why pain is related to selfinjury.

\section{Opioids}

'Endogenous opioids' are chemicals produced by our bodies as a natural painkiller when we experience pain. This can produce a 'natural-high', which may then become associated with selfinjury if this is felt after a child self-injures. This may increase the chances that the self-injurious behaviour will persist. ${ }^{65,66}$ Medications that block the effect of these opioids reduce some types of self-injury but not all, suggesting that this explanation may be limited to specific types of selfinjury. ${ }^{67,68}$

\section{Reduced pain perception}

It has been suggested that in some people reduced perception of pain might reduce the cost usually associated with self-injury, i.e. the pain caused by self-injury. ${ }^{65,66}$ Self-injurious behaviour can be shown by a child if the behaviour is reinforced (see Glossary section on page 20), for example by an adult giving attention to the child when they start to self-injure (e.g. by telling them to stop or asking if they are ok or by removing an uninteresting or challenging task). If pain is reduced then the cost is reduced but the 'gains' in terms of reward, from attention or task removal for example, remain the same. This is described as the behaviour having increased 'response efficiency' (see Glossary section on page 21) and is thus more likely to occur in the future.

Importantly however, evidence suggesting reduced pain perception has not been found in all people who show self-injury. For example pain-related facial expressions in response to instances of pain did not differ between people who showed self-injury and people who did not show self-injury ${ }^{64}$ This suggests that some individuals who show self-injury do not have reduced pain perception and so self-injury cannot be explained by a reduced sensitivity to pain in these people. ${ }^{66,65}$.

\section{'Gate control'}

Self-injury can stimulate non-pain receptors which affect the transmission of impulses travelling from pain receptors. This reduces the resulting pain experience. ${ }^{69,70,71}$ Children with intellectual disability may learn an association between stimulation around the site of pain and the resultant reduction of discomfort. These non self-injurious actions originally associated with reducing the perceived level of pain (for example rubbing an area) could then be shaped into self-injurious behaviours through learning processes (reinforcement, described above). However, in many cases the site of self-injury may not be where the pain is. Accounting for this, some people think that low level stimulation increases the threshold at which pain is perceived and that in 
individuals with intellectual disability who have pre-existing pain, more intense stimulation (such as self-injury), may be needed to decrease the perception of pain. ${ }^{72}$ This latter theory provides an explanation for the findings that the site of self-injury may not be where the pain is.

\section{'Setting events/establishing operations' (see Glossary section page 20)}

This is something that influences the relationship between the things that precede a behaviour, the behaviour itself and what happens after the behaviour that might reward it. For example, the presence of pain could increase the negative nature of a task demand (just as a frustrating situation becomes more irritating when we have a headache) and so make the removal of the demand more rewarding. Self-injury, previously rewarded by the removal of demanding tasks, would then be more likely to occur when pain was present. ${ }^{73,11,74}$

\section{How might pain be related to other challenging behaviours?}

We have seen how pain might cause self-injurious behaviour but most of these explanations do not appear to be able to explain other behaviours such as aggression or property destruction. An explanation which does seem able to account for the association between pain and aggressive behaviour or destruction of property is the theory that pain acts as a setting event/ establishing operation (see Glossary section on page 20). This explanation is described in detail above in relation to self-injury and is similar to the increased frustration we feel with everyday aversive situations when we are in pain. There is evidence consistent with this explanation; researchers have found that pain and discomfort during menstruation was associated with aggressive behaviour in the presence of demands relating to completing tasks (e.g. brushing teeth). ${ }^{74}$

It is important to remember that pain is often not the only factor influencing challenging behaviour, behaviours can have multiple functions (see Glossary section on page 20). Also a behaviour may begin by being related to pain but persist because of the learning processes described above.

\section{How can I tell what is causing my child's pain?}

If you suspect that your child is in pain it is important to find out what is causing the pain.

If there are no clear, visible causes of pain or discomfort or obvious illnesses (temperature, constipation) then it is important to rule out other physical causes with a check-up by a medical professional. This could include checking for signs of tooth decay/infection, reflux, ear infections and any other specific health problems suggested by your child's medical history or associated with a particular syndrome or disorder that they have.

In some cases children's behaviour may provide 'clues' as to the cause of their pain. For example self-injury may be targeted at locations associated with chronic pain. 
Examples of this include jaw punching if there is toothache, slapping the side of the head if there is earache and scratching the chest if there is reflux.

However it is certainly not the case that self-injury is always directed to the site of the pain, a child may head bang when in pain from constipation for instance. Alice's story in 'Focus on: pain and self-injury' (see below) is an example of self-injury (hair pulling) that is not directed at the supposed site of pain (gastric pain caused by reflux).

\section{Focus on: Pain and self-injury}

This case study describes how self-injurious behaviour may result from a person experiencing pain caused by a health condition which is common in the syndrome that they have. It brings together many of the themes identified throughout this briefing.

Alice's story: Alice was a young person with Cornelia de Lange syndrome who was showing a specific self-injurious behaviour: hair pulling. This behaviour concerned her caregivers and because of this they decided to take part in a research study of self-injury in people with genetic syndromes.

Observing Alice's behaviour: A research team carried out direct observations of Alice's selfinjury, planning to visit her on two occasions. The first time they met her she was awaiting a repeat prescription of her reflux medication. The research team observed her a number of times in different situations throughout the day. On this first visit to see Alice she showed self-injury during about $90 \%$ of the observations. This behaviour was quite severe, as in well over half of the occasions that the research team were observing her she pulled her hair between $10 \%-35 \%$ of the time.

Treatment for painful conditions: Between the visits Alice was given medical treatment which included reflux medication and dental treatment with two temporary fillings. 


\section{Graph showing Alice's behaviour before and after treatment for a painful}

health condition

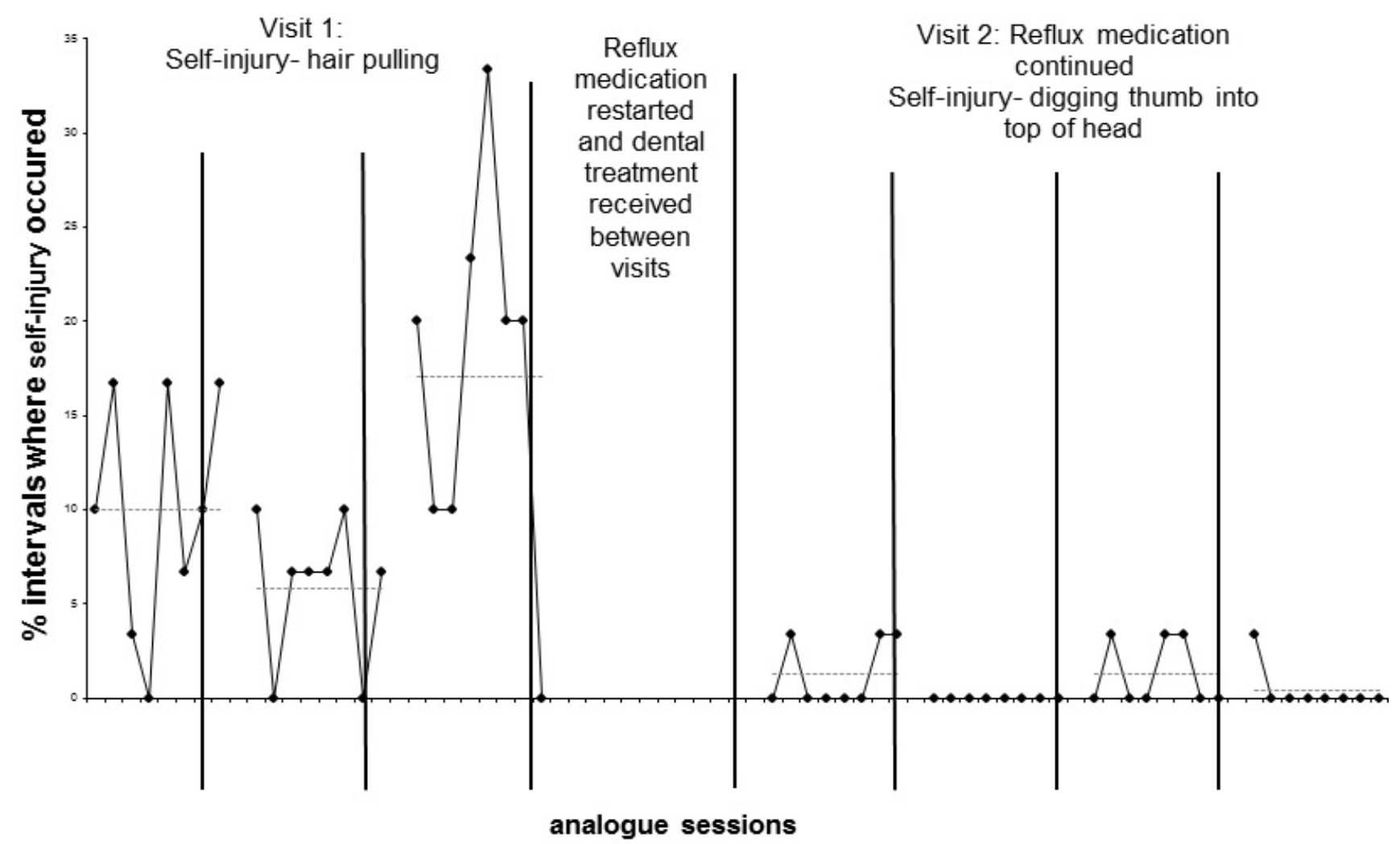

Change in behaviour after treatment: the next month after her dental treatment, and while she was on her medication that reduced stomach acid production, the researchers returned and again observed her behaviour. During this visit she showed no hair pulling at any point and showed no self-injury at all in $75 \%$ of observations. When she did show self-injury it lasted for less than $5 \%$ of the observations. Some self-injury (pressing her thumb onto the top of her head) was observed however.

\section{Key points:}

- Severe behaviour problems related to untreated pain can be addressed if the painful health conditions are treated.

- Self-injury may not always be directed towards the site of the pain - there is not a clear link between hair pulling and the pain caused by reflux (see 'Focus on: Gastro-oesophageal reflux' for more information about reflux on page 6).

- Some difficult behaviours may remain even after pain has been treated. 
Is the importance of identifying and treating pain in children with intellectual
disability widely recognised?

The importance of identifying and treating pain is not as widely recognised as it should be. The case has been made throughout this briefing that pain can have a negative impact on the lives of children with intellectual disability and that there needs to be specific attention to identifying and treating pain in these children. In the last ten years there have been a number of reports published which have supported this viewpoint.

Published reports: An independent inquiry launched in response to the 'Death by Indifference' report by Mencap found evidence of 'a significant level of avoidable suffering due to untreated ill-health' in people with intellectual disability. ${ }^{76}$ This neglect within the Health Service is purported to be the consequence of poor training and a lack of valid and reliable health and pain assessment tools. ${ }^{9}$

The 'Six lives: the provision of public services' report by the Parliamentary and Health Service Ombudsman (see Resources section on page 22) commented on 'unnecessary distress and suffering' of individuals with intellectual disability in the health service (page 10 of the report). It stated that 'the understanding of the issues [relating to health and social care in respect of people with intellectual disabilities] is at best patchy and at worst an indictment of our society' (page 30 of the report).

These reports further support the case for increased attention to the impact of pain in children with intellectual disability who are unable to report their pain.

\section{Key points:}

- If you feel that your child is experiencing pain which is not being treated because they are unable to express this, this must be addressed.

- It is important that they receive the medical treatment that is their right and that caregivers are able to access this on their behalf.

- Increasing awareness of the causes, signs and impact of pain is a step towards addressing this issue. 
Aggression: Non-accidental behaviours initiated by an individual towards another individual that cause the other individual harm or have the potential to cause them harm (e.g. kicking, hitting).

Autism Spectrum Disorder: A condition that affects social interaction, communication, interests and behaviour. Behaviours shown by individuals with Autism Spectrum Disorder include problems with social interaction and communication, and restricted and repetitive patterns of thought, interests and physical behaviours.

Baseline: A measurement taken of 'typical' functioning before any changes occur or are made.

Gastro-oesophageal reflux disorder: A disorder caused by stomach acid leaking from the stomach up into the oesophagus (the tube from the mouth to the stomach), potentially causing discomfort and pain.

Function/s: What a behaviour 'does' for the person who is showing it, e.g. when a child shows self-injury when presented with a demanding task and the task is then removed, the self-injury is said to have the function of escaping a task demand.

Makaton: A programme which uses signs and symbols, together with spoken language to aid communication.

Pain signature: The unique pattern of behaviours that a person may show when they are in pain.

PECS (Picture Exchange Communication System): A programme which uses pictures to aid communication. At the beginning individuals give a picture of a desired item in exchange for the item and then this is built on to teach more complex communication.

Property destruction: Non-accidental behaviour which causes damage to the person's environment.

Reinforced: A behaviour is reinforced when there is a response to it which acts as a reward of some kind which makes the behaviour more likely. For example when a child experiences social contact as rewarding, its presence following self-injury may lead to reinforcement.

Establishing operation/setting event: A broader factor influencing difficult behaviour (e.g. selfinjury, aggression) by changing the relationship between a behaviour and the consequence. An example of this is hunger; if a child's aggressive behaviour is rewarded by a caregiver providing a snack to distract them, if they are hungry (the establishing operation, the broader factor) then the behaviour (aggression) is more likely to occur. Hunger has changed the relationship between the behaviour (aggression) and the response (provision of food) so that this response becomes more rewarding to the person. 
Repetitive \& stereotyped behaviour: A wide range of behaviours which are characterised by frequency of repetition (how often they are repeated), inappropriateness (whether they are inappropriate to the context/setting they are shown in) and invariance (lack of change in the behaviours shown). Such behaviours might include body rocking, hand flapping, lining up or organising objects, repetitive questioning.

Respiratory tract infection: An infection which affects the sinuses, throat, airways or lungs.

Response efficiency: How efficient a behaviour is (for the person showing it) at eliciting a response. This depends on how much effort the person has to put into the behaviour, how often the behaviour is reinforced, how quickly it is reinforced and how rewarding the reinforcing response is. A behaviour has high response efficiency if it does not require a lot of effort from the person doing it, it is rewarded often or quickly or is rewarded with a highly desired response.

Self-injurious behaviour/self-injury: Non-accidental behaviours initiated by a person which cause that individual harm (e.g. head banging, hand biting, scratching).

Self-report: A type of measure or assessment that is completed by the person who is being assessed.

Vocalisations: Sounds made by an individual. For example laughing, shouting, screaming, grunting. 


\section{Resources}

\section{Cerebra research briefings/infographic}

To find the following research briefings and infographic visit Cerebra's research section on the website:

http://w3.cerebra.org.uk/research

- Self-injurious behaviour in children with an intellectual disability

- Is the diagnosis of a genetic disorder important for children with intellectual disability?

- FLACC PainScale

\section{Online guidance}

NHS information about heartburn and gastro-oesophageal reflux disease

http://www.nhs.uk/Conditions/Gastroesophageal-reflux-disease/Pages/Introduction.aspx

NICE guidelines for the prevention and management of pressure sores

https://www.nice.org.uk/guidance/cg179/chapter/1-recommendations\#/prevention-neonatesinfants-children-and-young-people

Mencap guidance on postural care

https://www.mencap.org.uk/posturalcare

\section{Online report}

Six Lives: the provision of public services to people with learning disabilities

http://www.ombudsman.org.uk/reports-and-consultations/reports/health/archived-reports/sixlives-the-provision-of-public-services-to-people-with-learning-disabilities

\section{Website presenting information about genetic syndromes}

Cerebra Centre for Neurodevelopmental Disorders FIND website (Further Inform Neurogenetic Disorders)

http://www.findresources.co.uk 


\section{References}

1. Van Schrojenstein Lantman-de Valk, H., Linehan, C., Kerr M., \& Noonan-Walsh P. (2007). Developing health indicators for people with intellectual disabilities. The method of the Pomona project. Journal of Intellectual Disability Research, 6, 427-434.

2. Emerson, E., \& Hatton, C. (2007a). Mental health of children and adolescents with intellectual disabilities in Britain. The British Journal of Psychiatry, 191(6), 493-499.

3. Emerson, E., \& Hatton, C. (2007b). Poverty, socio-economic position, social capital and the health of children and adolescents with intellectual disabilities in Britain: a replication. Journal of Intellectual Disability Research, 51(11), 866-874.

4. Puri, B. K., Lekh, S. K., Langa, A., Zaman, R., \& Singh, I. (1995). Mortality in a hospitalized mentally handicapped population: a 10-year survey. Journal of Intellectual Disability Research, 39(5), 442446.

5. Lewis, C.W. (2009). Dental Care and Children with Special Health Care Needs: A PopulationBased Perspective. Academic Pediatrics, 9 (6), 420-426.

6. Tiller, S., Wilson, K. I., \& Gallagher, J. E. (2001). Oral health status and dental service use of adults with learning disabilities living in residential institutions and in the community. Community Dental Health, 18(3), 167-171.

7. Bowley, C., \& Kerr, M. (2000). Epilepsy and intellectual disability. Journal of Intellectual Disability Research, 44(5), 529-543.

8. Howells, G. (1986). Are the medical needs of mentally handicapped adults being met?. The Journal of the Royal College of General Practitioners, 36(291), 449-453.

9. Malviya, S., Voepel-Lewis, T., Merkel, S., \& Tait, A. R. (2005). Difficult pain assessment and lack of clinician knowledge are ongoing barriers to effective pain management in children with cognitive impairment. Acute Pain, 7(1), 27-32.

10. Emerson, E., Baines, S., Allerton, L., \& Welch, V. (2010). Health inequalities and people with learning disabilities in the UK: 2010. Durham: Improving Health \& Lives: Learning Disabilities Observatory.

11. Carr, E. G., \& Blakeley-Smith, A. (2006). Classroom intervention for illness-related problem behavior in children with developmental disabilities. Behavior modification, 30(6), 901-924.

12. Bosch,J., Van Dyke, D.C., Milligan Smith,S.,\& Poulton, S. (1997). 'Role of Medical Conditions in the Exacerbation of Selfinjurious Behavior: An Exploratory Study', Mental Retardation, 35 (2), 124-130. 
13. Hartman, E. C., Gilles, E., McComas, J. J., Danov, S. E., \& Symons, F. J. (2008). Clinical observation of self-injurious behavior correlated with changes in scalp morphology in a child with congenital hydrocephalus. Journal of child neurology, 23(9), 1062-1065.

14. Taylor, D.V., Rush, D., Hetrick, W.P., \& Sandman, C.A. (1993). Self-injurious behavior within the menstrual cycle of women with mental retardation. American Journal on Mental Retardation, 97(6), 659-664.

15. Böhmer, C.J.M., Niezen-de Boer, M.C., Klinkenberg-Knol, E. C., Devillé, W.L.J.M., Nadorp, J.H.S.M., \& Meuwissen, S.G.M. (1999). The prevalence of gastroesophageal reflux disease in institutionalized intellectually disabled individuals. The American journal of gastroenterology, 94(3), 804-810.

16. Böhmer, C. J. M., Taminiau, J. A. J. M., Klinkenberg-Knol, E. C., \& Meuwissen, S. G. M. (2001). The prevalence of constipation in institutionalized people with intellectual disability. Journal of Intellectual Disability Research, 45(3), 212-218.

17. Clayton, J., \& Smith, M.M. (1993). Clinical research on Angelman syndrome in the United Kingdom: observations on 82 affected individuals. American Journal of Medical Genetics, 46, 1215.

18. Kline, A. D., Krantz, I. D., Sommer, A., Kliewer, M., Jackson, L. G., FitzPatrick, D. R., Levin, A., \& Selicorni, A. (2007). Cornelia de Lange syndrome: clinical review, diagnostic and scoring systems, and anticipatory guidance. American journal of medical genetics part A, 143(12), 1287-1296.

19. Luzzani, S., Macchini, F., Valade, A., Milani, D., \& Selicorni, A. (2003). Gastroesophageal reflux and Cornelia de Lange syndrome: typical and atypical symptoms. American Journal of Medical Genetics Part A, 119(3), 283-287.

20. Wilkins, L. E., Brown, J. A., Nance, W. E., \& Wolf, B. (1983). Clinical heterogeneity in 80 homereared childrenwith cri du chat syndrome. The Journal of pediatrics, 102(4), 528-533.

21. Horvath, K., Papadimitriou, J.C., Rabsztyn, A., Drachenberg, C., \& Tildon, J.T. (1999). Gastrointestinal abnormalities in children with autistic disorder. Journal of Pediatrics, 135, 559-563.

22. Butler, J. V., Whittington, J. E., Holland, A. J., Boer, H., Clarke, D., \& Webb, T. (2002). Prevalence of, and risk factors for, physical ill-health in people with Prader-Willi syndrome: A population-based study. Developmental Medicine \& Child Neurology, 44(4), 248-255.

23. Sellier, E., Uldall, P., Calado, E., Sigurdardottir, S., Torrioli, M. G., Platt, M. J., \& Cans, C. (2012). Epilepsy and cerebral palsy: Characteristics and trends in children born in 1976-1998. European Journal of Paediatric Neurology, 16(1), 48-55.

24. Cooper, S. A., Smiley, E., Jackson, A., Finlayson, J., Allan, L., Mantry, D., \& Morrison, J. (2009). Adults with intellectual disabilities: prevalence, incidence and remission of aggressive behaviour and related factors. Journal of Intellectual Disability Research, 53(3), 217-232. 
25. Oliver, C., Murphy, G, H., \&Corbett, J, A. (1987) Self-injurious behaviour in people with learning disabilities: Determinants and interventions. International Review of Psychiatry, 2, 101-116.

26. McClintock K., Hall S. \& Oliver C. (2003). Risk markers associated with challenging behaviours in people with intellectual disabilities: a meta-analytic study. Journal of Intellectual Disabilities Research 47, 405-416.

27. Poppes, P., Van der Putten, A. J. J., \& Vlaskamp, C. (2010). Frequency and severity of challenging behaviour in people with profound intellectual and multiple disabilities. Research in Developmental Disabilities, 31(6), 1269-1275.

28. Arron, K., Oliver, C., Moss, J., Berg, K., \& Burbidge, C. (2011). The prevalence and phenomenology of self-injurious and aggressive behaviour in genetic syndromes. Journal of Intellectual Disability Research, 55(2), 109-120.

29. Hyman, P., Oliver, C., \& Hall, S. (2002). Self-injurious behavior, self-restraint, and compulsive behaviors in Cornelia de Lange syndrome. American Journal on Mental Retardation, 107(2).

30. Oliver, C., Sloneem, J., Hall, S., \& Arron, K. (2009). Self-injurious behaviour in Cornelia de Lange syndrome: 1 . Prevalence and phenomenology. Journal of Intellectual Disability Research, 53(7), 575-589.

31. Baghdadli, A., Pascal, C., Grisi, S., \& Aussilloux, C. (2003). Risk factors for self-injurious behaviours among 222 young children with autistic disorders. Journal of Intellectual Disability Research, 47(8), 622-627.

32. Schopler, E., Reichler, R. J., DeVellis, R. F., \& Daly, K. (1980). Toward objective classification of childhood autism: Childhood Autism Rating Scale (CARS). Journal of Autism and Developmental Disorders, 10(1), 91-103.

33. Petty, J. and Oliver, C. (2005). Self-injurious behaviour in people with intellectual disability. Current Opinion in Psychiatry, 18, 484-489.

34. Deb, S., Thomas, M., \& Bright, C. (2001). Mental disorder in adults with intellectual disability. 2: The rate of behaviour disorders among a community-based population aged between 16 and 64 years. Journal of Intellectual Disability Research, 45(6), 506-514.

35. Ross, E. \& Oliver, C. (2002). The relationship between levels of mood, interest and pleasure and "challenging" behaviour in adults with severe and profound intellectual disabilities. Journal of Intellectual Disability Research, 46, 191-197.

36. Smiley, E., \& Cooper, S. A. (2003). Intellectual disabilities, depressive episode, diagnostic criteria and Diagnostic Criteria for Psychiatric Disorders for Use with Adults with Learning Disabilities/ Mental Retardation (DC-LD). Journal of Intellectual Disability Research, 47(1), 62-71. 
37. Finucane, B., Dirrigl, K. H., \& Simon, E. W. (2001). Characterization of self-injurious behaviors in children and adults with Smith-Magenis syndrome. American Journal on Mental Retardation, 106(1).

38. Greenberg M. A., Wortman C. B., Stone A. A. (1996). Emotional expression and physical health Revising traumatic memories or fostering self regulation. Journal of Personality and Social Psychology71, 588-602.

39. Martin, S. C., Wolters, P. L., \& Smith, A. C. (2006). Adaptive and maladaptive behavior in children with Smith-Magenis syndrome. Journal of Autism and Developmental Disorders, 36(4), 541-552.

40. Friedenberg, F. K., Xanthopoulos, M., Foster, G. D., \& Richter, J. E. (2008). The association between gastroesophageal reflux disease and obesity. The American journal of gastroenterology, 103(8), 2111-2122.

41. Dogra, H., Lad, B., \& Sirisena, D. (2011). Paediatric gastro-oesophageal reflux disease. Young Children, 6, 10.

42. Traube, M. (1990). The spectrum of the symptoms and presentations of gastroesophageal reflux disease. Gastroenterology clinics of North America, 19(3), 609-616.

43. Crino, P. B., Nathanson, K. L., \& Henske, E. P. (2006). The tuberous sclerosis complex. New England Journal of Medicine, 355(13), 1345-1356.

44. Clarke, A., Hancock, E., Kingswood, C., \& Osborne, J. (1994). Nephrology Dialysis. Transplantation, 14, 988-991.

45. Curatolo, P., Bombardieri, R., \& Jozwiak, S. (2008). Tuberous sclerosis. The Lancet, 372(9639), 657-668.

46. Leung, A. K., \& Robson, W. L. M. (2007). Tuberous sclerosis complex: a review. Journal of Pediatric Health Care, 21(2), 108-114.

47. Chu-Shore, C. J., Major, P., Camposano, S., Muzykewicz, D., \& Thiele, E. A. (2010). The natural history of epilepsy in tuberous sclerosis complex. Epilepsia, 51(7), 1236-1241.

48. Joinson, C., O'Callaghan, F. J., Osborne, J. P., Martyn, C., Harris, T., \& Bolton, P. F. (2003). Learning disability and epilepsy in an epidemiological sample of individuals with tuberous sclerosis complex. Psychological medicine, 33(02), 335-344.

49. Roach, E. S., DiMario, F. J., Kandt, R. S., \& Northrup, H. (1999). Tuberous sclerosis consensus conference: recommendations for diagnostic evaluation. Journal of child neurology, 14(6), 401407. 
50. Berg, K., Arron, K., Burbidge, C., Moss, J., \& Oliver, C. (2007). Carer-Reported Contemporary Health Problems in People With Severe and Profound Intellectual Disability and Genetic Syndromes. Journal of Policy and Practice in Intellectual Disabilities, 4(2), 120-128.

51. Merkel, S.I., Voepel-Lewis, T., Shayevitz, J.R., \& Malviya, S. (1997). The FLACC: A behavioral scale for scoring postoperative pain in young children. Pediatric Nursing, 23(3), 293- 297.

52. Voepel-Lewis, T., Merkel, S., Tait, A. R., Trzcinka, A., \& Malviya, S. (2002). The reliability and validity of the Face, Legs, Activity, Cry, Consolability observational tool as a measure of pain in children with cognitive impairment. Anesthesia \& Analgesia, 95(5), 1224-1229.

53. Malviya, S., Voepel-Lewis, T. E. R. R. I., Burke, C., Merkel, S., \& Tait, A. R. (2006). The revised FLACC observational pain tool: improved reliability and validity for pain assessment in children with cognitive impairment. Pediatric Anesthesia, 16(3), 258-265.

54. Breau, L., McGrath, P, Finley, A., \& Camfield, C. (2004). The Non-Communicating Children's Pain Checklist- Revised. Nova Scotia, Canada.

55. Carr, E. G., \& Owen-DeSchryver, J. S. (2007). Physical illness, pain, and problem behavior in minimally verbal people with developmental disabilities. Journal of Autism and Developmental Disorders, 37(3), 413-424.

56. Breau, L. M., McGrath, P. J., Camfield, C. S., \& Finley, G. A. (2002). Psychometric properties of the non-communicating children's pain checklist-revised. Pain, 99(1), 349-357.

57. Stinson, J. N., Kavanagh, T., Yamada, J., Gill, N., \& Stevens, B. (2006). Systematic review of the psychometric properties, interpretability and feasibility of self-report pain intensity measures for use in clinical trials in children and adolescents. Pain, 125(1), 143-157.

58. Fanurik, D., Koh, J. L., Harrison, R. D., Conrad, T. M., \& Tomerun, C. (1998). Pain assessment in children with cognitive impairment an exploration of self-report skills. Clinical Nursing Research, 7(2), 103-119.

59. Benini, F., Trapanotto, M., Gobber, D., Agosto, C., Carli, G., Drigo, P., Eland, J., \& Zacchello, F. (2004). Evaluating pain induced by venipuncture in pediatric patients with developmental delay. The Clinical Journal of Pain, 20(3), 156-163.

60. Breau, L. M., \& Burkitt, C. (2009). Assessing pain in children with intellectual disabilities. Pain Research \& Management: The Journal of the Canadian Pain Society, 14(2), 116.

61. Ekman, P., \& Friesen, W. V. (1978). Facial Action Coding System: A technique for the measurement of facial movement. Palo Alto: Consulting Psychologists Press. 
62. Courtemanche, A., Schroeder, S., Sheldon, J., Sherman, J., \& Fowler, A. (2012). Observing signs of pain in relation to self-injurious behaviour among individuals with intellectual and developmental disabilities. Journal of Intellectual Disability Research, 56(5), 501-515.

63. Symons, F. J., \& Danov, S. E. (2005). A prospective clinical analysis of pain behavior and selfinjurious behavior. Pain, 117(3), 473-477.

64. Symons, F. J., Harper, V. N., McGrath, P. J., Breau, L. M., \& Bodfish, J. W. (2009). Evidence of increased non-verbal behavioral signs of pain in adults with neurodevelopmental disorders and chronic self-injury. Research in Developmental Disabilities, 30(3), 521-528.

65. Rojahn, J., Schroeder, S. R., \& Hoch, T. A. (2007). Self-injurious behavior in intellectual disabilities (Vol. 2). Elsevier.

66. Sandman, C.A. (1990/1991). The opiate hypothesis in autism and self-injury. Journal of Child and Adolescent Psychopharmacology 1 (3), 237-248.

67. Symons, F. J. (2011). Self-injurious behavior in neurodevelopmental disorders: relevance of nociceptive and immune mechanisms. Neuroscience \& Biobehavioral Reviews, 35(5), 1266-1274.

68. Symons, F. J., Thompson, A., \& Rodriguez, M. C. (2004). Self-injurious behavior and the efficacy of naltrexone treatment: A quantitative synthesis. Mental Retardation and Developmental Disabilities Research Reviews, 10(3), 193-200.

69. Melzack, R., Wall, P, D. (1965) Pain Mechanisms: a new theory. Science, 150, 971-979.

70. Melzack, R., Wall, P. D., \& Ty, T. C. (1982). Acute pain in an emergency clinic: latency of onset and descriptor patterns related to different injuries. Pain, 14(1), 33-43.

71. Woolf, C.J., \& Slater, M.W. (2000) Neuronal plasticity: increasing the gain in pain. Science, 288, 1765-1769.

72. Peebles, K. A., \& Price, T. J. (2012). Self-injurious behaviour in intellectual disability syndromes: evidence for aberrant pain signalling as a contributing factor. Journal of Intellectual Disability Research, 56(5), 441-452.

73. Carr, E. G., \& Smith, C. E. (1995). Biological setting events for self-injury. Mental Retardation and Developmental Disabilities Research Reviews, 1(2), 94-98.

74. Carr, E. G., Smith, C. E., Giacin, T. A., Whelan, B. M., \& Pancari, J. (2003). Menstrual discomfort as a biological setting event for severe problem behavior: Assessment and intervention. American Journal on Mental Retardation, 108(2). 
75. Breau, L.M., Camfield, C., Symons, F.J., Bodfish, J.W., Mackay, A., Finley, G.A., \& McGrath, P.J. (2003). Relation between pain and self-injurious behavior in nonverbal children with severe cognitive impairments. The Journal of pediatrics, 142, 498-503.

76. Michael, J., \& Richardson, A. (2008). Healthcare for all: the independent inquiry into access to healthcare for people with learning disabilities. Tizard Learning Disability Review, 13(4), 28-34.

\section{Authors}

Dr. Lucy Wilde, Cerebra Centre for Neurodevelopmental Disorders, School of Psychology, University of Birmingham, Birmingham, UK

Lucy Wilde is a Postdoctoral Research Fellow at the University of Birmingham, working with Professor Chris Oliver in the Cerebra Centre for Neurodevelopmental Disorders. She completed her PhD on the behavioural and cognitive phenotype of Smith-Magenis syndrome at the University of Birmingham. Her research interests include social functioning in neurodevelopmental disorders, inhibition deficits and emotional control and factors influencing challenging behaviour in people with intellectual disability.

\section{Dr. Kate Eden, University of Bath}

Kate Eden conducted her PhD at the Cerebra Centre for Neurodevelopmental Disorders, under the supervision of Professor Chris Oliver. Her PhD focussed on the association between pain and challenging behaviour in children with intellectual disability. Following completion of her $\mathrm{PhD}$, she continued to work at the Cerebra Centre as a research fellow contributing to an ongoing research project collecting longitudinal data from families of children with rare genetic syndromes. She is now completing a doctorate in Clinical Psychology at the University of Bath.

\section{Prof. Chris Oliver, Cerebra Centre for Neurodevelopmental Disorders, School of Psychology, University of Birmingham, Birmingham, UK}

Chris Oliver is Professor of Neurodevelopmental Disorders at the University of Birmingham and director of the Cerebra Centre for Neurodevelopmental Disorders. He trained as a clinical psychologist at Edinburgh University before completing a PhD on self-injurious behaviour in people with intellectual disability at the Institute of Psychiatry, London. He is currently researching early intervention, behaviour disorders in people with severe intellectual disability and autism spectrum disorder, behavioural phenotypes in genetic syndromes and neuropsychological and behavioural assessment for people with severe intellectual disability. He has published over 100 peer reviewed articles in scientific journals, is Editor in Chief for the Journal of Intellectual Disability Research and serves on a number of scientific advisory committees for syndrome support groups. Sadly, he supports Luton Town Football Club. 


\section{Reviewer}

\section{Dr. Caroline Richards, Cerebra Centre for Neurodevelopmental Disorders, School of}

Psychology, University of Birmingham, Birmingham, UK

Caroline Richards is a Research Fellow and Clinical Psychologist at the Cerebra Centre for Neurodevelopmental Disorders, University of Birmingham. She has previously conducted research investigating the prevalence, risk markers and function of self-injurious behaviour in children and adults with autism spectrum disorder. She has additional research interests in the profile of autism spectrum disorder in rare genetic syndromes and is currently conducting research assessing sleep difficulties in children with neurodevelopmental disorders. 